\title{
Beyond Local Appearance: Category Recognition from Pairwise Interactions of Simple Features
}

\author{
Marius Leordeanu ${ }^{1} \quad$ Martial Hebert $^{1} \quad$ Rahul Sukthankar $^{2,1}$ \\ mleordealandrew.cmu.edu heberteri.cmu.edu rahulsecs.cmu.edu \\ ${ }^{1}$ Carnegie Mellon University ${ }^{2}$ Intel Research Pittsburgh
}

\begin{abstract}
We present a discriminative shape-based algorithm for object category localization and recognition. Our method learns object models in a weakly-supervised fashion, without requiring the specification of object locations nor pixel masks in the training data. We represent object models as cliques of fully-interconnected parts, exploiting only the pairwise geometric relationships between them. The use of pairwise relationships enables our algorithm to successfully overcome several problems that are common to previously-published methods. Even though our algorithm can easily incorporate local appearance information from richer features, we purposefully do not use them in order to demonstrate that simple geometric relationships can match (or exceed) the performance of state-of-the-art object recognition algorithms.
\end{abstract}

\section{Introduction}

Object category recognition is very challenging because there is no formal definition of what constitutes an object category. While people largely agree on common, useful categories, it is still not clear which are the objects' features that help us group them into such categories. Our proposed approach is based on the observation that for a wide variety of common object categories, shape matters more than local appearance. For example, it is the shape, not the color or texture, that enables a plane to fly, an animal to run or a human hand to manipulate objects. Many categories are defined by their function and it is typically the case that function dictates an object's shape rather than its low level surface appearance. In this paper we represent these object category models as cliques of very simple features (sparse points and their normals), and focus only on the pairwise geometric relationships between them.

The importance of using pairwise geometric constraints between simple shape features in object recognition was identified early on based on the observation that accidental alignments between oriented edges are rare and that they can be effective in pruning the search for correspondences between sets of model and input features. This is consistent with research in cognitive science hypothesizing that human category recognition is based on pairwise relationships between object parts [13]. In computer vision, this has led to the development of several promising approaches, such as interpretation trees, or alignment and verification. While successful, these techniques were limited by combinatorial issues (see [12] for a survey of early work in this area). All of these techniques were limited by their reliance on an explicit, parametric, representation of the transformation between model and input data (e.g., rigid or affine). To avoid the need for combinatorial search, global techniques that integrate pairwise geometric constraints into a global energy were also proposed (e.g., using MRF models [18]). Later approaches relaxed all of these limitations by using a nonparametric model of deformation between model data and input data. This is the case of the work of Berg et al. [2], which uses second-order geometric relationships between features in a global optimization framework.

Our approach combines many of these ideas from early work and more recent contributions. We explicitly represent the pairwise relations between contour elements by using a large set of parameters, in contrast to the pairwise distance and angle used in previous work [2]. We show that we can achieve good recognition performance, without using local appearance features (such as those used by Berg et al.), validating earlier observations [12] that geometric constraints alone can prune most correspondences.

We also formulate the search for consistent correspondences as a global energy optimization, with two key differences from earlier work. First, we use an efficient algorithm for finding an optimal discrete set of consistent correspondences [17], which enable us to use a large number of features. Second, we learn the parameters used in representing the geometric constraints in order to better capture the space of pairwise deformations.

Our matching-based approach builds a single abstract shape model that incorporates common features from multiple training images, as well as distinctive characteristics unique to each training image. Applying this in a semi- 
supervised learning context enables us to automatically discover and remove the irrelevant clutter from training images, keeping only the features that are indeed useful for recognition. This gives us a more compact representation, which reduces the computational and memory cost, and improves generalization.

An interesting alternative to geometric matching is to classify directly based on statistics of the spatial distribution of features $[10,24,26]$. In contrast to these fully-supervised approaches, we show that that simple geometric relationships can be successful in a semi-supervised setting.

The use of second-order geometric relationships enables our algorithm to successfully overcome problems often encountered by other methods:

1. During training, the algorithm is translation invariant, robust to clutter, and does not require aligned training images. This is in contrast with previous work such as $[2,11,22,23]$.

2. We efficiently learn models consisting of hundreds of fully interconnected parts (capturing both short- and long-range dependencies). Most previous work handles models only up to 30 sparsely inter-connected parts, such as the star-shaped [6,9], k-fan, [7] or hierarchical models $[4,8]$. There has been work [5] handling hundreds of parts, but such models are not translationinvariant and each object part is connected only to its k-nearest neighbors.

3. We select features based on how well they work together as a team rather than individually (as is the case in [7, 23]). This gives us a larger pool of useful features, which are collectively discriminative, if not necessarily on an individual basis.

We formulate the problem as follows: given a set of negative images (not containing the object) and weakly-labeled positive images (containing an object of a given category somewhere in the image), the task is to learn a category shape model (Section 3) that can be used both for the localization and recognition of objects from the same category in novel images (Section 2). This problem is challenging because we do not have any prior knowledge about the object's location in the training images. Also these images can contain a substantial amount of clutter that is irrelevant to the category that we want to model. All that is known at training time is that the object is present somewhere in the positive training images and absent in the negative ones.

\section{The Category Shape Model}

The category shape model is a graph of interconnected parts (nodes) whose geometric interactions are modeled using pairwise potentials inspired by Conditional Random Fields $[14,15]$. The nodes in this graph are fully interconnected (they form a clique) with a single exception: there is no link between two parts that have not occurred together in the training images. These model parts have a very sim-

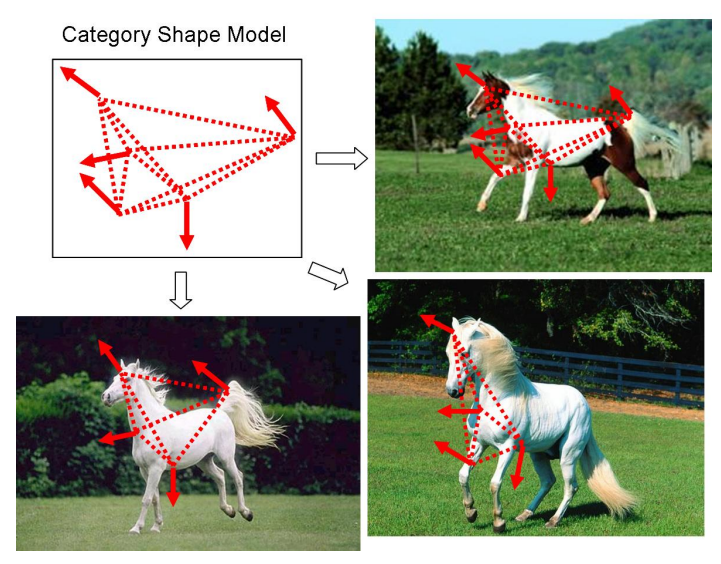

Figure 1. The model is a graph whose edges are abstract pairwise geometric relationships. It integrates generic configurations common to most objects from a category as well as more specific configurations that capture different poses and aspects.

ple representation: they consist of sparse, abstract points together with their associated normals. Of course we could add local information in addition to their normals, but our objective is to assess the power of the geometric relationships between these simple features.

We represent the pairwise relationships by an overcomplete set of parameters. The parts as well as their geometric relationships are learned from actual boundary fragments extracted from training images. The model is a graph whose edges are abstract pairwise geometric relationships. It is a compact representation of a category shape, achieved by sharing generic geometric configurations common to most objects from a category and also by integrating specific configurations that capture different aspects or poses (Figure 1).

This section first describes the structure of the model, including, in particular, the details of the pairwise geometric relations, by showing how model parts are matched with features from an input image, which we term object localization. Then, given correspondences between model parts and image features, we show how to estimate the likelihood that the input image will contain the desired category, i.e., the recognition problem. Here we assume that a model has been learned from training data, the details of the learning procedure are described in Section 3.

\subsection{Object Localization}

We define the object localization problem as finding which feature in the image best matches each model part. We formulate it as a quadratic assignment problem (QAP), which incorporates the second-order relationships. The matching score $E$ is written as:

$$
E_{x}=\sum_{i a ; j b} x_{i a} x_{i b} G_{i a ; j b}
$$




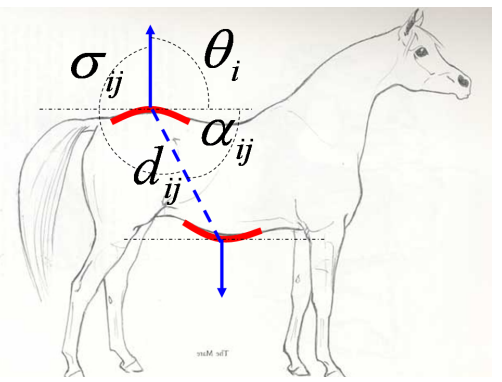

Figure 2. Parameters that capture the pair-wise geometric relationships between object parts

Here $x$ is an indicator vector with an entry for each pair $(i, a)$ such that $x_{i a}=1$ if model part $i$ is matched to image feature $a$ and 0 otherwise. With a slight abuse of notation we consider $i a$ to be a unique index for the pair $(i, a)$. We also enforce the mapping constraints that one model part can match only one model feature and vice versa: $\sum_{i} x_{i a}=$ 1 and $\sum_{a} x_{i a}=1$.

The pairwise potential $G_{i a ; j b}$ (terminology borrowed from graphical models) reflects how well the parts $i$ and $j$ preserve their geometric relationship when being matched to features $a, b$ in the image. Similar to previous approaches taken in the context of CRFs [14] we model these potentials using logistic classifiers :

$$
G_{i a ; j b}=\frac{1}{1+\exp \left(-\mathbf{w}^{T} \mathbf{g}_{i j}(a, b)\right)}
$$

Here $\mathbf{g}_{i j}(a, b)$ is a vector describing the geometric deformations between the parts $(i, j)$ and their matched features $(a, b)$. We now explain in greater detail the type of features used and their pairwise relationships. As mentioned already, each object part can be seen as an abstract point and its associated normal (with no absolute location). For a pair of model parts $(i, j)$ we capture their translation-invariant relationship in the vector $\mathbf{e}_{i j}=$ $\left\{\theta_{i}, \theta_{j}, \sigma_{i j}, \sigma_{j i}, \alpha_{i j}, \beta_{i j}, d_{i j}\right\}$, where $d_{i j}$ represents the distance between them, $\beta_{i j}$ is the angle between their normals and the rest are angles described in Figure 2.

The same type of information is extracted from input images, each image feature corresponding to a point sampled from some boundary fragment extracted from that image (see Section 5). We consider a similar pairwise relationship $\mathbf{e}_{a b}$ for the pair $(a, b)$ of image features that were matched to $(i, j)$. Then we express the pairwise geometric deformation vector as $\mathbf{g}_{i j}(a, b)=\left[1, \epsilon_{1}^{2}, \ldots, \epsilon_{7}^{2}\right]$, where $\epsilon=\mathbf{e}_{i j}-\mathbf{e}_{a b}$. Notice that the geometric parameters $\mathbf{e}_{i j}$ form an over-complete set of values, some highly dependent on each other. Considering all of them becomes very useful for geometric matching and recognition because it makes $G_{i a ; j b}$ more robust to changes in the individual elements of $\mathbf{g}_{i j}(a, b)$.

In order to localize the object in the image, we find the assignment $\mathrm{x}^{*}$ that maximizes the matching score $E$ (written in matrix notation by setting $\left.\mathbf{G}(i a ; j b)=G_{i a ; j b}\right)$ :

$$
\mathbf{x}^{*}=\operatorname{argmax}\left(\mathbf{x}^{T} \mathbf{G} \mathbf{x}\right)
$$

For one-to-one constraints (each model part can match only one image feature and vice-versa) this combinatorial optimization problem is known as the quadratic assignment problem (QAP). For many-to-one constraints it is also known in the graphical models literature as MAP inference for pairwise Markov networks. In general, both problems are intractable. We enforce the one-to-one constraints and use the spectral matching algorithm [16], which is very efficient in practice, giving good approximate solutions and being able to handle hundreds of fully connected parts in a few seconds on a $2 \mathrm{GHz}$ desktop computer.

\subsection{Discriminative Object Recognition}

The previous section describes how we localize the object by efficiently solving a quadratic assignment problem. However, this does not solve the recognition problem since the matching algorithm will return an assignment even if the input image does not contain the desired object. In order to decide whether the object is present at the location $\mathrm{x}^{*}$ specified by our localization step, we model the posterior $P\left(C \mid \mathbf{x}^{*}, D\right)$ (where the class $C=1$ if the object is present at location $\mathrm{x}^{*}$ and $C=0$ otherwise). Modeling the true posterior would require modeling the likelihood of the data $D$ given the background category (basically, the rest of the world), which is infeasible in practice. Instead, we take a discriminative approach and attempt to model this posterior directly, as described below.

We consider that $P\left(C \mid \mathbf{x}^{*}, D\right)$ should be a function of several factors. First, it should depend on the quality of the match (localization) as given by the pairwise potentials $G_{i a ; j b}$ for the optimal solution $\mathrm{x}^{*}$. Second, it should depend only on those model parts that indeed belong to the category of interest and are discriminative against the negative class. It is not obvious which are those parts, since we learn the model in a semi-supervised fashion. For this reason we introduce the relevance parameter $r_{i}$ for each part $i$ (Section 3 explains how this is learned), which has a high value if part $i$ is discriminative against the background, and low value otherwise. We approximate the posterior with the following logistic classifier:

$$
S\left(\mathbf{G}_{o}, \mathbf{r}\right)=\frac{1}{1+\exp \left(-q_{0}-q_{1} \sigma(\mathbf{r})^{T} \mathbf{G}_{\mathbf{o}} \sigma(\mathbf{r})\right)} .
$$

The matrix $\mathbf{G}_{\mathbf{o}}(i, j)=G_{i a^{*} ; j b^{*}}$ contains all the pairwise potentials for the optimal localization $\mathrm{x}^{*}$. The rows and columns of $\mathbf{G}_{\mathbf{o}}$ corresponding to model parts not found in the image are set to 0 . In Eqn. (4), each pairwise potential $G_{i j}$ is weighted by the product $\sigma\left(r_{i}\right) \sigma\left(r_{j}\right)$, where $\sigma\left(r_{i}\right)=$ $1 /\left(1+e^{-r_{i}}\right)$.

The primary reason for passing the relevance parameters through a sigmoid function is the following: letting the relevances be unconstrained real-valued parameters would 


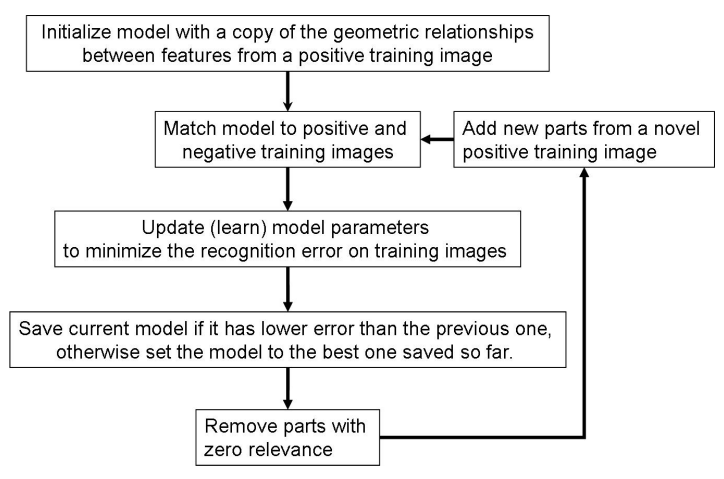

Figure 3. Learning algorithm overview

not help us conclusively establish which parts indeed belong to the category and which ones do not. What we really want is a binary relevance variable that is 1 if the model part belongs to the category model and 0 otherwise. Having a binary variable would allow us to consider only those parts that truly belong to the object category and discard the irrelevant clutter. Our intuition is that if we squash the unconstrained relevances $r_{i}$ we effectively turn them into soft binary variables, and during training we force them to be either relevant $\left(\sigma\left(r_{i}\right) \approx 1\right)$ or irrelevant $\left(\sigma\left(r_{i}\right) \approx 0\right)$. This is exactly what happens in practice. The squashed relevances of most parts go either to 1 or 0 , thus making it possible to remove the irrelevant ones $\left(\sigma\left(r_{i}\right) \approx 0\right)$ without affecting the approximate posterior $S\left(\mathbf{G}_{\mathbf{o}}, \mathbf{r}\right)$. An additional benefit of squashing the relevance parameters is that it damps the effect of very large or very small negative values of $r_{i}$, reducing overfitting without the need for an explicit regularization term.

The higher the relevances $r_{i}$ and $r_{j}$, the more $\mathbf{G}_{\mathbf{o}}(i, j)$ contributes to the posterior. It is important to note that the relevance of one part is considered with respect to its pairwise relationships with all other parts together with their relevances. Therefore, parts are evaluated based on how well they work together as a team, rather than individually. Finally, an important aspect of the approach is that we interpret the logistic classifier $S\left(\mathbf{G}_{\mathbf{o}}, \mathbf{r}\right)$ not as the true posterior, which is impractical to compute, but rather as a distance function that is specifically tuned for classification.

\section{Learning}

The model parameters to be learned consist of: the pairwise geometric relationships $\mathbf{e}_{i j}$ between all pairs of parts, the sensitivity to deformations $\mathbf{w}$ (which defines the pairwise potentials), the relevance parameters $\mathbf{r}$ and $q_{0}, q_{1}$ (which define the classification function $S$ ). The learning steps are summarized in Figure 3 and detailed below.

\subsection{Initialization}

We first initialize the pairwise geometric parameters $\left(\mathbf{e}_{i j}\right)$ for each pair of model parts by simply copying them from a positive training image. Thus, our initial model will have as many parts as the first training image used and the same pairwise relationships. We initialize the rest of the parameters to a set of default values. For each part $i$ we set the default value of its $r_{i}$ to $0\left(\sigma\left(r_{i}\right)=0.5\right)$. The default parameters of the pairwise potentials (w) are learned independently as described in Section 4.

\subsection{Updating the Parameters}

Starting from the previous values, we update the parameters by minimizing the familiar sum-of-squares error function (typically used for training neural networks) using sequential gradient descent. The objective function is differentiable with respect to $\mathbf{r}, q_{0}$ and $q_{1}$ since they do not affect the optimum $\mathrm{x}^{*}$ (for the other parameters we differentiate assuming fixed $\mathrm{x}^{*}$ ):

$$
J=\sum_{n=1}^{N} b_{n}\left(S\left(\mathbf{G}_{\mathbf{o}}{ }^{(n)}, r\right)-t^{(n)}\right)^{2} .
$$

Here $t^{(n)}$ denotes the ground truth for the $n^{\text {th }}$ image ( 1 if the object is present in the image, 0 otherwise). The weights $b_{n}$ are fixed to $m_{N} / m_{P}$ if $t^{(n)}=1$ and 1 otherwise, where $m_{N}$ and $m_{P}$ are the number of negative and positive images, respectively. These weights balance the relative contributions to the error function between positive and negative examples. The matrix $\mathbf{G}_{\mathbf{o}}{ }^{(n)}$ contains the pairwise potentials for the optimal localization for the $n^{t h}$ image.

We update the parameters using sequential gradient descent, looping over all of the training images for a fixed number of iterations; in practice this reliably leads to convergence. The learning update for any given model parameter $\lambda$ for the $n^{\text {th }}$ example has the general form of:

$$
\lambda \leftarrow \lambda-\rho b_{n}\left(S\left(\mathbf{G}_{\mathbf{o}}{ }^{(n)}, \mathbf{r}\right)-t^{(n)}\right) \frac{\partial S\left(\mathbf{G}_{\mathbf{o}}{ }^{(n)}, \mathbf{r}\right)}{\partial \lambda},
$$

where $\rho$ denotes the learning rate. Using this general rule we can easily write the update rules for all of the model parameters. The pairwise potentials $\left(\mathbf{G}_{\mathbf{0}}\right)$ do not depend on the parameters $\mathbf{r}, q_{0}, q_{1}$. It follows that the optimal labeling $\mathrm{x}^{*}$ of the localization problem remains constant if we only update $\mathbf{r}, q_{0}, q_{1}$. In practice we only update $\mathbf{r}, q_{0}, q_{1}$ and the pairwise distances $d_{i j}$, while assuming that $\mathrm{x}^{*}$ does not change, thus avoiding the computationally-expensive step of matching after each gradient descent update.

\subsection{Removing Irrelevant Parts}

As mentioned earlier, the relevance values $\sigma\left(r_{i}\right)$ for each part $i$ tend to converge either toward 1 or 0 . This is due to the fact that the derivative of $J$ with respect to the 

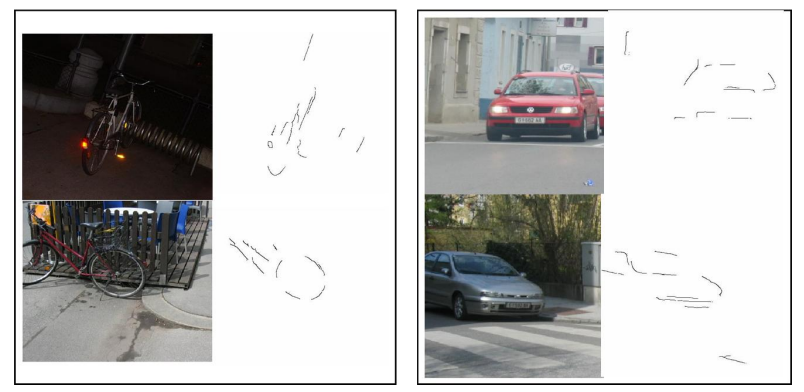

Figure 4. The model integrates geometric configurations belonging to different aspects (view-points) within the same category. Training images (left) and the boundary fragments containing the relevant parts learned from different view-points and integrated in the same model (right) are shown for the bike and car categories. Note that the algorithm automatically determines the features that belong to the object rather than the background, despite the fact that the object appears in very different aspects in the training set.

free relevance parameters $r_{i}$ is zero only when the output $S\left(\mathbf{G}_{\mathbf{o}}{ }^{(n)}, \mathbf{r}\right)$ is either 0 or 1 , or when the relevance $\sigma\left(r_{i}\right)$ is either 0 or 1 , the latter being much easier to achieve. This is the key factor that allows us to discard irrelevant parts without significantly affecting the output $S\left(\mathbf{G}_{\mathbf{o}}{ }^{(n)}, \mathbf{r}\right)$. Therefore, all parts with $\sigma\left(r_{i}\right) \approx 0$ are discarded. In our experiments we observe that the relevant features typically belong to the true object of interest (Figure 5).

\subsection{Adding New Parts}

We proceed by merging the current model with a newlyselected training image (randomly selected from the ones on which the recognition output is not close enough to 1 ): we first localize the current model in the new image, thus finding the subset of features in the image that shares similar pairwise geometric relationships with the current model. Next, we add to the model new parts corresponding to all of the image features that fail to match the current model parts. As before, we initialize all the corresponding parameters involving newly-added parts by copying the geometric relationships between the corresponding features and using default values for the rest. At this stage, different view-points or shapes of our category can be merged (Figure 4). The geometric configurations shared by different aspects are already in the model (that is why we first perform matching) and only the novel configurations are added (from the parts that did not match). After adding new parts we return to the stage of updating the parameters (Figure 3 ). We continue this loop until we are satisfied with the error rate.

The approach of adding training images one-by-one is related to incremental semi-supervised learning methods [25]. In our case, we later discard the information that is not useful for recognition (the parts with zero relevance). Removing and adding parts enables our model to grow or shrink dynamically, as needed for the recognition task.
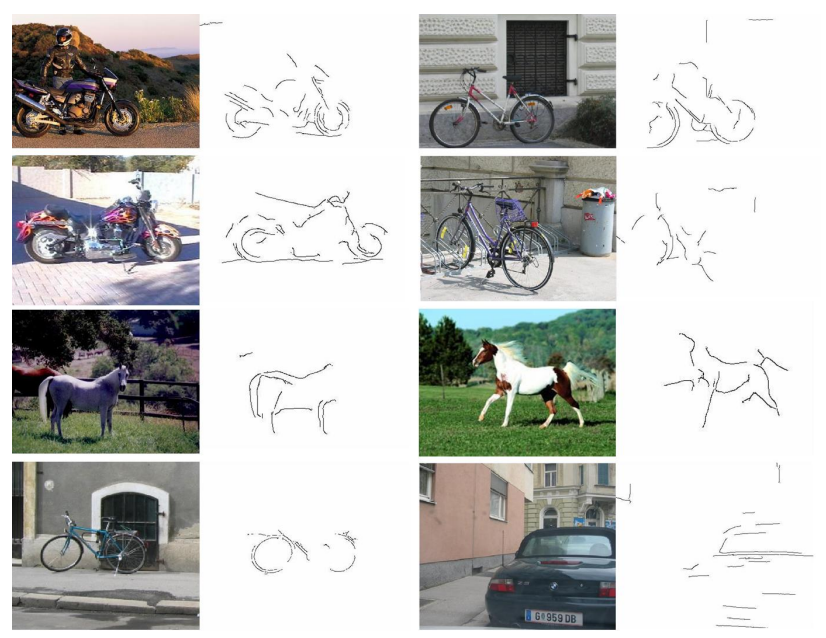

Figure 5. Training images (Left) and the contours on which the relevant features were found during training (Right).

\section{Learning Pairwise Geometric Potentials}

We learn a default set of parameters $\mathbf{w}$ for the pairwise potentials independently of the object parts $(i, j)$ and the object class $C$. Learned independently, the pairwise potentials are logistic classifiers designed to model the posterior that a given pair of assignments is correct given the geometric deformation $g$, regardless of the object class $C$ or the specific parts $(i, j)$. We learn the default $\mathbf{w}$, from a set of manually-selected correct correspondences and randomly-selected set of incorrect ones, using the iteratively re-weighted least-squares algorithm. The correspondences are selected from different databases used in the literature: CALTECH-5 (faces, motorbikes, airplanes, motorcycles, cars, leaves, background), INRIA-horses and GRAZ-02 (person, bikes, cars, background). The same set of default $\mathbf{w}$ is used in all of our recognition experiments.

Data points $\mathbf{g}_{i j}(a, b)$ are collected for both the positive (pair of correct correspondences) and the negative class (at least one assignment is wrong), where image feature $a$ from one image is matched to the image feature $i$ from the other image. For randomly-selected pairs of images containing the same object category, we manually select approximately 8000 pairs of correct correspondences per database (whenever the poses were similar enough to enable finding exact correspondences between the contours of the two images). We select 16000 pairs of wrong correspondences per database.

Table 1 shows how the geometry-based pairwise classifier generalizes across different object categories. This pairwise classifier operates on pairs of assignments only and it is not a classifier of object categories. In particular, this set of experiments quantifies the extent to which pairwise geometric constraints can be used to distinguish between correct and incorrect correspondences, but it does not say anything about the effectiveness of the constraints to distinguish between categories. In this set of experiments we 
Table 1. The classification rates (at equal error rate) of the geometry-based pairwise classifier trained and tested on different databases. Results are averaged over 10 runs. The numbers measure the discrimination between correct and incorrect correspondences, not the category recognition performance.

\begin{tabular}{|l|r|r|r|}
\hline Database & $\begin{array}{r}\text { Caltech-5 } \\
\text { (train) }\end{array}$ & $\begin{array}{r}\text { INRIA } \\
\text { (train) }\end{array}$ & $\begin{array}{r}\text { GRAZ-02 } \\
\text { (train) }\end{array}$ \\
\hline \hline Caltech-5 (test) & $97.42 \%$ & $97.65 \%$ & $97.23 \%$ \\
\hline INRIA (test) & $94.66 \%$ & $95.33 \%$ & $94.31 \%$ \\
\hline GRAZ-02 (test) & $92.93 \%$ & $93.73 \%$ & $93.24 \%$ \\
\hline
\end{tabular}

train the classifier on pairs of candidate assignments from one database and test it on pairs of assignments from all three databases. We repeat this ten times for different random splits of the training and testing sets and average the results. The interesting fact is that the performance of the classifier is roughly the same (within 1\%) for a given test database (indexed by row), regardless of which database was used for training (indexed by column). This strongly suggests that the same classifier is learned each time, which further implies that the space of geometric second-order deformations is more or less the same for a large variety of solid objects under similar imaging conditions. It follows that the pairwise geometry can be used with confidence on a wide variety of objects even with a single set of default parameters. Our recognition experiments actually use the default parameters learned only from the Caltech-5 database. Table 1 supports the hypothesis that accidental alignments are rare events regardless of the object class.

As a side-experiment we also investigate the performance of the pairwise geometric classifier $G_{i j}(a, b)$ against that of a pairwise classifier that only uses local features such as SIFT [19], shape context [1] and textons [21] extracted at the same locations. As before, positive and negative vectors for pairs of correspondences are collected - this time using changes in local feature descriptors rather than geometry. More precisely the pairwise appearance changes are represented by $\mathbf{f}_{i j}(a, b)=\left[1,\left\|s_{i}-s_{a}\right\|^{2},\left\|s_{j}-s_{b}\right\|^{2}, \| c_{i}-\right.$ $\left.c_{a}\left\|^{2},\right\| c_{j}-c_{b}\left\|^{2},\right\| t_{i}-t_{a}\left\|^{2},\right\| t_{j}-t_{b} \|^{2}\right]$. Here $s_{i}, c_{i}$ and $t_{i}$ represent the SIFT, Shape Context and the 32 texton histogram descriptors, respectively, at the location of feature $i$. Using the logistic regression algorithm we train appearanceonly classifiers as well as classifiers combined with geometry (using the vectors $\left.\left[\mathbf{f}_{i j}(a, b), \mathbf{g}_{i j}(a, b)\right]\right)$. As before, note that these classifiers are independent of the object category. Their only task is to classify a specific pair of correspondences $((i, a),(j, b))$ as correct/wrong.

Table 2 presents comparisons among the performances of the three types of classifiers (geometry, appearance and combined geometry+appearance). For each database we randomly split the pairs of correspondences in 6000 training (2000 positive and 4000 negative) and 18000 test (6000 positive and 10000 negative) vectors. An interesting observa-
Table 2. The classification rates (at equal error rate) of the geometry-based pairwise classifier vs. the local feature classifier, for three different databases, averaged over 10 runs. Note that these are not category recognition results, but the results of classifiers on pairs of assignments.

\begin{tabular}{|l|r|r|r|}
\hline Database & local only & geometry only & combined \\
\hline \hline Caltech-5 & $86.73 \%$ & $97.42 \%$ & $98.10 \%$ \\
\hline INRIA horses & $80.89 \%$ & $95.33 \%$ & $96.40 \%$ \\
\hline GRAZ-02 & $83.30 \%$ & $93.24 \%$ & $94.74 \%$ \\
\hline
\end{tabular}

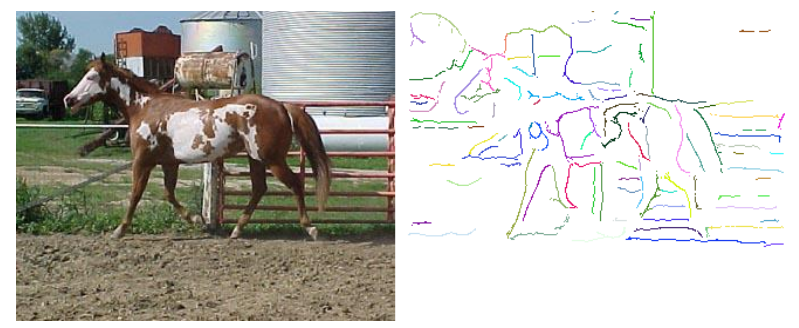

Figure 6. Original image (left) and extracted contours (right).Right: different colors correspond to different connected components.

tion is that, in each case the geometry-only classifier outperforms the one based on local features by at least $10 \%$. This could be attributed to the fact that, while object shape remains relatively stable within the same category, object appearance varies significantly. Moreover, combining appearance and geometry only improves performance marginally $(1-1.5 \%)$ over the geometry-only classifier. These results validate our approach of focusing on second-order relationships between simple shape features rather than on richer individual local features.

\section{Implementation Details: Grouping Edges into Contours}

We first obtain the edge map by using the $P b$ edge detector from Martin et al. [21]. Next, we remove spurious edges using a grouping technique inspired by Mahamud et $a l$. [20]. Then we select image features by evenly sampling them (at every 20 pixels) along edge contours, as shown in Figure 6. Since the $P b$ detector is rather expensive, we also experimented with using a Canny edge detector instead and we obtain similar results after grouping the edge elements into connected contour fragments.

We obtain these contours by grouping pixels that form long and smooth curves. First, we group the edges into connected components by joining those pairs of edge pixels $(i, j)$ that are both sufficiently close (i.e., within 5 pixels) and satisfy smoothness constraints based on collinearity and proximity (thus ensuring that the components only contain smooth contours).

For each connected component we form its weighted ad- 
jacency matrix A such that $\mathbf{A}(i, j)$ is positive if edge pixels $(i, j)$ are connected and 0 otherwise. The value of $\mathbf{A}(i, j)$ increases with the smoothness between $(i, j)$. The principal eigenvalue $\lambda$ of $\mathbf{A}$ describes the average smoothness of this component. We keep only those components that are large enough (number of pixels $>$ sizeThresh) and smooth enough $(\lambda>$ smoothThresh $)$.

This step is very efficient, usually taking less than a second per image, in Matlab on a $2 \mathrm{GHz}$ PC. The main use of these contours is to eliminate spurious edges more reliably than by simply thresholding the output of the edge detector. It also provides a better estimate of the normal at each edge pixel by considering only neighboring edges belonging to the same contour (Figure 6).

\section{Experiments}

Tables 3 and 4 compare the performance of our method with Winn et al. [27] on the Pascal challenge training dataset $^{1}$ (587 images). This is an interesting experiment because our method only employs geometry and ignores local appearance; in contrast, Winn et al. focus on local texture information, while ignoring the geometry. We follow the same experimental setup, splitting the dataset randomly in two equal training and testing sets. The first set of experiments uses the provided bounding box (also used by Winn et al.). We outperform the texture-based classifier by more than $10 \%$, confirming our intuition that shape is a stronger cue than local appearance for these types of object categories. Surprisingly, bikes and motorcycles are not confused as much as one might expect despite their similar shapes. In the second set of experiments, we do not use the bounding boxes, ${ }^{2}$ neither for training nor testing, in order to demonstrate that our method's ability to learn in a weakly-supervised setting. The performance drops by approximately $5 \%$, which is significant, but relatively low considering that the objects of interest in this experiment frequently occupy only a small fraction of the image area. A more serious challenge is that several positive images for one class contain objects from other categories (e.g., there are people present in some of the motorcycle and car images). In our reported resuls, an image from the "motorcycle" class containing both a motorbike and a person that was classified as "person" would be treated as an error.

As mentioned earlier, the models are compact representations of the relevant features present in the positive training set. The algorithm discovers relevant parts that, in our experiments, generally belong to the true object of interest despite significant background clutter. An interesting and useful feature of our method is its ability to integrate different view-points, aspects or shapes within the same category

\footnotetext{
${ }^{1}$ http://www.pascal-network.org/challenges/VOC/voc2005/. We ignore the gray-level UIUC car images.

${ }^{2}$ For the few images in which the object was too small, we select a bounding box of 4 times the area of the original bounding box.
}

Table 3. Confusion Matrix on Pascal Dataset.

\begin{tabular}{|l|r|r|r|r|}
\hline Category & Bikes & Cars & Motorbikes & People \\
\hline \hline Bikes & $80.7 \%$ & $0 \%$ & $7 \%$ & $12.3 \%$ \\
\hline Cars & $5.7 \%$ & $88.6 \%$ & $5.7 \%$ & $0 \%$ \\
\hline Motorbikes & $4.7 \%$ & $0 \%$ & $95.3 \%$ & $0 \%$ \\
\hline People & $7.1 \%$ & $0 \%$ & $0 \%$ & $92.9 \%$ \\
\hline
\end{tabular}

Table 4. Average multiclass recognition rates on Pascal.

\begin{tabular}{|c|c|c|c|}
\hline Algorithm & $\begin{array}{c}\text { Ours } \\
\text { (bbox) }\end{array}$ & $\begin{array}{c}\text { Ours } \\
\text { (no bbox) }\end{array}$ & $\begin{array}{c}\text { Winn } \text { et al. } \\
\text { (bbox) }\end{array}$ \\
\hline \hline Pascal Dataset & $89.4 \%$ & $84.8 \%$ & $76.9 \%$ \\
\hline
\end{tabular}

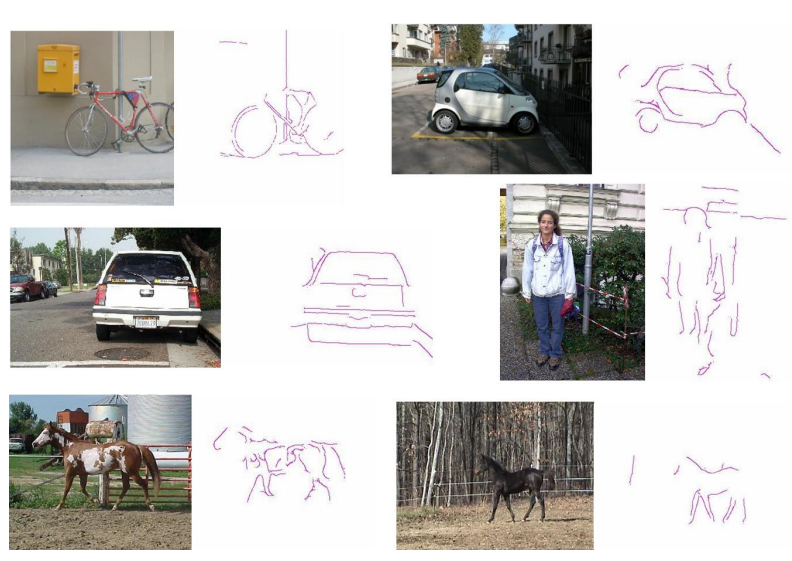

Figure 7. Training Images (Left) and the contours on which the relevant features were found (Right)

(Figure 4). This happens automatically, as new parts are added from positive images.

The computational cost of classifying a single image does not depend on the number of training images: the model is a compact representation of the relevant features in the training images, usually containing between 40 to 100 parts. The size of the model is not fixed manually; it is an automatic outcome from the learning stage.

We also compare our method with Opelt et al. [23] on the GRAZ-01 and GRAZ-02 datasets (Table 5). We run the experiments on the same training and test sets on full images (no bounding boxes were used). Opelt et al. focus mainly on local appearance and select descriptors based on their individual performance and combine them using AdaBoost. This is in contrast with our approach, since our features by themselves have no discriminative power. It is their combined configuration that makes them together discriminative.

We further test our algorithm on the INRIA (168 images) and Weizmann Horse (328 images) [3] databases, using for the negative class the GRAZ-02 background images. We do not use objects masks (nor bounding boxes) and we randomly split the images in equal training and testing sets. 
Table 5. Category recognition rates (at equal error rate) on GRAZ Dataset (People and Bikes), Shotton and INRIA horses datasets. Bounding boxes (masks) are not used.

\begin{tabular}{|l|r|r|r|}
\hline Dataset & Ours & $\begin{array}{r}\text { Opelt } \text { et al. } \\
(1)\end{array}$ & $\begin{array}{r}\text { Opelt } \text { et al. } \\
(2)\end{array}$ \\
\hline \hline People (GRAZ I) & $82.0 \%$ & $76.5 \%$ & $56.5 \%$ \\
\hline Bikes (GRAZ I) & $84.0 \%$ & $78.0 \%$ & $83.5 \%$ \\
\hline People (GRAZ II) & $86.0 \%$ & $70.0 \%$ & $74.1 \%$ \\
\hline Bikes (GRAZ II) & $92.0 \%$ & $76.4 \%$ & $74.0 \%$ \\
\hline Horses (Shotton) & $92.02 \%$ & - & - \\
\hline Horses (INRIA) & $87.14 \%$ & - & - \\
\hline
\end{tabular}

The INRIA horse database includes significant changes in scale, pose and multiple horses present in the same image.

\section{Conclusion}

We demonstrate that exploiting the pairwise interactions between simple shape features enables us to match and even exceed the performance of state-of-the-art algorithms that use more complex descriptors. Our results confirm the intuition that shape is a very powerful cue for object category recognition. This paper demonstrates that by capturing shape through second-order relationships (as opposed to local, first-order descriptors), we can build flexible models that accommodate significant deformations, while still being discriminative against background clutter.

\section{Acknowledgments}

This research was performed in part for the Intelligent Robotics Development Program, one of the 21st Century Frontier R\&D Program funded by the Ministry of Commerce, Industry and Energy of Korea; and in part by a summer internship at Intel Research Pittsburgh.

\section{References}

[1] S. Belongie, J. Malik, and J. Puzicha. Shape context: A new descriptor for shape matching and object recognition. In NIPS, 2000.

[2] A. Berg, T. Berg, and J. Malik. Shape matching and object recognition using low distortion correspondences. In $E C C V$, 2006.

[3] E. Borenstein, E. Sharon, and S. Ullman. Combining topdown and bottom-up segmentation. In CVPR, 2004.

[4] G. Bouchard and B. Triggs. Hierarchical part-based visual object categorization. In CVPR, 2005.

[5] G. Carneiro and D. Lowe. Sparse flexible models of local features. In $E C C V, 2006$.

[6] D. Crandall, P. Felzenszwalb, and D. Huttenlocher. Spatial priors for part-based recognition using statistical models. In CVPR, 2005.
[7] D. Crandall and D. Huttenlocher. Weakly supervised learning of part-based spatial models for visual object recognition. In $E C C V, 2006$.

[8] P. Felzenszwalb and D. Huttenlocher. Pictorial structures for object recognition. IJCV, 2004.

[9] R. Fergus, P. Perona, and A. Zisserman. A sparse object category model for efficient learning and exhaustive recognition. In $C V P R, 2005$.

[10] V. Ferrari, L. Fevrier, F. Jurie, and C. Schmid. Groups of adjacent contour segments for object detection. Technical report, INRIA, 2006.

[11] V. Ferrari, T. Tuytelaars, and L. V. Gool. Object detection by contour segment networks. In ECCV, 2006.

[12] W. E. L. Grimson. Object Recognition by Computer: The Role of Geometric Constraints. MIT Press, 1990.

[13] J. E. Hummel. Where view-based theories break down: The role of structure in shape perception and object recognition. In Cognitive Dynamics: Conceptual Change in Humans and Machines. Erlbaum, 2000.

[14] S. Kumar. Models for Learning Spatial Interactions in Natural Images for Context-Based Classification. PhD thesis, The Robotics Institute, Carnegie Mellon University, 2005.

[15] J. D. Lafferty, A. McCallum, and F. C. N. Pereira. Conditional random fields: Probabilistic models for segmenting and labeling sequence data. In ICML, 2001.

[16] M. Leordeanu and M. Hebert. A spectral technique for correspondence problems using pairwise constraints. In ICCV, 2005.

[17] M. Leordeanu and M. Hebert. Efficient map approximation for dense energy functions. In ICML, May 2006.

[18] S. Li. Markov random field modeling in computer vision. Springer-Verlag, Cambridge, 1995.

[19] D. Lowe. Distinctive image features from scale-invariant keypoints. IJCV, 60(4), 2004.

[20] S. Mahamud, L. R. Williams, K. K. Thornber, and K. Xu. Segmentation of multiple salient closed contours from real images. PAMI, April 2003.

[21] D. Martin, C. Fowlkes, and J. Malik. Learning to detect natural image boundaries using local brightness, color, and texture cues. PAMI, May 2004.

[22] K. Mikolajczyk, B. Leibe, and B. Schiele. Multiple object class detection with a generative model. In CVPR, 2006.

[23] A. Opelt, A. Pinz, M. Fussenegger, and P. Auer. Generic object recognition with boosting. PAMI, March 2006.

[24] A. Opelt, A. Pinz, and A. Zisserman. A boundary-fragmentmodel for object detection. In ECCV, 2006.

[25] C. Rosenberg. Semi-Supervised Training of Models for Appearance-Based Statistical Object Detection Methods. $\mathrm{PhD}$ thesis, Carnegie Mellon University, 2004.

[26] J. Shotton, A. Blake, and R. Cipolla. Contour-based learning for object detection. In ICCV, 2005.

[27] J. Winn, A. Criminisi, and T. Minka. Object categorization by learned universal visual dictionary. In ICCV, 2005 . 